\title{
Autophagy localization and cytoprotective role in cisplatin-induced acute kidney injury
}

\author{
Shanika Karunasagara, Geum-Lan Hong, Da-Young Jung, Si-Yun Ryu*, Ju-Young Jung* \\ Department of Veterinary Medicine \& Institute of Veterinary Science, College of Veterinary Medicine, \\ Chungnam National University, Daejeon 34134, Korea
}

\begin{abstract}
Autophagy is a fundamental cellular process that maintains homeostasis and cell integrity, under stress conditions. Although the involvement of autophagy in various conditions has been elucidated, the role of autophagy in renal structure is not completely clarified. Our aim was to investigate the cytoprotective effect of autophagy against acute kidney injury (AKI) through cisplatin deteriorative pathway, which leads to AKI via renal cell degradation. For in vivo experiments, male Sprague Dawley rats were divided in to 2 groups ( $n=6 /$ group) as control, Cis-5D. Following a single intraperitoneal injection of cisplatin, rats were sacrificed after 5 days. Blood urea nitrogen (BUN), creatinine (Cr) and histological alterations were examined. Further, expression of key regulators of autophagy, light-clain 3 (LC3), p62, and Beclin1, was evaluated by immunohistochemistry (IHC). The rats exhibited severe renal dysfunction, indicated by elevated BUN, Cr. Hematoxylin and eosin staining revealed histological damages in cisplatintreated rats. Furthermore, IHC analysis revealed increased expression of LC3, Beclin1 and decreased expression of p62. Furthermore, expression of aforementioned autophagy markers was restricted to proximal tubule. Taken together, our study demonstrated that cisplatin can cause nephrotoxicity and lead to AKI. This phenomenon accelerated autophagy in renal proximal tubules and guards against AKI.
\end{abstract}

Keywords: acute kidney injury; autophagy; cisplatin, LC3

*Corresponding author

Ju-Young Jung

Department of Veterinary Medicine \& Institute of Veterinary Science, College of Veterinary

Medicine, Chungnam National University, 99

Daehak-ro, Yuseung-gu, Daejeon 34134, Korea

Tel: +82-42-821-8899, 7902

Fax: +82-42-821-8899

E-mail: jyjung@cnu.ac.kr

Si-Yun Ryu

Department of Veterinary Medicine \& Institute of Veterinary Science, College of Veterinary

Medicine, Chungnam National University, 99

Daehak-ro, Yuseung-gu, Daejeon 34134, Korea

Tel: +82-42-821-7903

Fax: +82-42-821-8899

E-mail: syryu@cnu.ac.kr

ORCID:

Shanika Karunasagara-

https://orcid.org/0000-0002-3049-5177

Geum-Lan Hong

Da-Young Jung

Si-Yun Ryu-

https://orcid.org/0000-0003-4235-4192

Ju-Young Jung-

https://orcid.org/0000-0002-6355-5738

Conflict of Interest

The authors declare no conflicts of interest.

Received: May 16, 2019

Revised : June 11, 2019

Accepted: June 28, 2019

\section{Introduction}

Nephrotoxicity is one of the major side effects of chemotherapy and accounts for up to $60 \%$ of all reported acute kidney injury (AKI) cases with a considerable mortality rate [1]. Several factors, such as oxidative stress, inflammation, mitochondrial dysfunction, and direct cytotoxicity to tubular epithelial cells, are responsible for the development of nephrotoxicity [2]. AKI by ischemia, sepsis, or nephrotoxins leads to conditions of renal stress, further leading to cell death, tissue damage, loss of renal function, and consequently kidney failure [3-7]. In the present study, we used cisplatin to induce nephrotoxicity to cause AKI.

Cisplatin (cis-diamminedichloroplatinum [II]; CDDP) is a platinum-containing anticancer drug widely use for the treatment of solid cancers, including breast, head, neck, lung, testis, and ovary [2]. However, cisplatin induces a number of toxicities, such as nephrotoxicity, gastrotoxicity, myelosuppression, ototoxicity, and allergic reactions. Furthermore, nephrotoxicity is considered as the major dose-dependent side effect [8]. According to previous reports, $20 \%$ of patients who receive high-dose cisplatin treatment suffer from renal dysfunction, whereas approximately one-third of patients present kidney injury after initial intervention [2]. Pathophysiological characteristics of cisplatin-induced kidney injury include induction of renal vasoconstriction, decrease in renal plasma flow, and reduction in glomerular filtration rate. Moreover, this condition may increase serum creatinine $(\mathrm{Cr})$ and reduce serum magnesium and potassium levels. In addition, it is conceived that cisplatin may cause permanent diminution of renal functions. The major pathological phenomena of cisplatin-induced nephrotoxicity are injury of kidney tubular cell and death [1].

Autophagy is an intracellular process that entails the transfer of cytoplasmic constituents to the lysosome to be degraded and recycled. This cellular process is up-regulated in various pathological and stress conditions to elimi- 
nate damaged components, thereby providing defense against the diseased condition. Although autophagy in kidney was first identified in 1970, clarifying the role of autophagy in kidney tubules is still in the nascent stage $[9,10]$. Previous investigations have reported that in nephrotoxic and septic AKI conditions, autophagy is vigorously induced in proximal tubular cells [11]. Further, deletion of genes, such as Atg5 and Atg7 related to autophagy, attenuates autophagy induction in AKI, thereby worsening kidney injury [12-14]. In addition, cisplatin takes part in mitochondrial dysfunction and increases the reactive oxygen species production in autophagy deficient renal proximal tubular cells, thereby leading to AKI $[15,16]$. However, it is still unclear how autophagy is activated in AKI, and further examinations are needed to determine the exact mechanism behind this process.

In the present study, we performed cisplatin-induced nephrotoxicity to evaluate AKI using histology and immunohistochemistry (IHC). We also studied the role of autophagy and its localization under AKI condition to evaluate the expression of key markers light-clain 3 (LC3), p62, and Beclin1. Moreover, we focused on the relationship between cisplatin treatment and autophagy progression.

\section{Materials and Methods}

\section{Experimental animals}

For this experiment, 4-week-old Sprague Dawley (SD) rats ( $\mathrm{n}=18$, purchased from www.orientbio.com) were used, and animals were fed with standard diet and water ad libitum under controlled temperature, humidity and lighting conditions $\left(22 \pm 2^{\circ} \mathrm{C}, 55 \pm 5 \%\right.$, and 12:12 light:dark cycle, respectively) throughout the experimental period. All experimental protocols regarding animals were approved by the International Ethics Committee at Chungnam National University (CNU-00068). After 7 days of acclimation, rats were divided in to 2 groups as control and Cis-5D. After single intraperitoneal injection of cisplatin ( $5 \mathrm{mg} / \mathrm{kg}$, Sigma, USA), except for the control group, rats were sacrificed after 5 days. Blood sample of each animal was analyzed at the beginning and end of the experimental period.

\section{Renal function analysis}

Blood urea nitrogen (BUN) and Cr levels were measured to evaluate the pathogenesis of acute renal failure. Blood samples were obtained from retro-orbital plexus of rats before and after ( 5 day) cisplatin treatment and incubated for $30 \mathrm{~min}$ in an ice-cold box before centrifugation at 2,000 $\times \mathrm{g}$ for $10 \mathrm{~min}$ at $4^{\circ} \mathrm{C}$ to collect the supernatant. Serum concentration of BUN and $\mathrm{Cr}$ were measured using an automatic blood chemical analyzer (IDEXX Laboratories, Inc., USA).

\section{Preservation of renal tissue}

Experimental animals were anesthetized via an intraperitoneal injection of tiletamine $(30 \mathrm{mg} / \mathrm{kg})$ plus zolazepam $(10$ $\mathrm{mg} / \mathrm{kg}$ ), and perfusion in kidneys was preserved through the abdominal aorta in vivo. The rats were initially perfused briefly with phosphate buffered saline (PBS; osmolality 298 mosmol/ $/ \mathrm{kg} \mathrm{H}_{2} \mathrm{O} ; \mathrm{pH}$ 7.4) to rinse all the blood, followed by perfusion with periodate lysine paraformaldehyde (PLP) solution for $5 \mathrm{~min}$. Next, kidneys were removed and cut into sagittal slices of 1-2 mm thickness and were overnight postfixed in PLP solution at $4{ }^{\circ} \mathrm{C}$. Tissues were dehydrated and embedded in wax (polyethylene glycol 400 disterate, Polysciences, USA). Then, the fixed slices were cut transversely at a thickness of $5 \mu \mathrm{m}$ using a microtome (Leica RM2135, Leica, Germany), and wax sections were stained with hematoxylin and eosin for histological examination and IHC studies.

\section{IHC analysis}

After dewaxing, sections were treated with $0.5 \%$ Triton $\mathrm{X}$ 100-PBS solution for $30 \mathrm{~min}$ and washed 3 times with PBS. Nonspecific binding sites were blocked with normal goat/ horse serum diluted to $1: 10$ in $0.3 \%$ PBS for 30-60 min, and incubated at $4{ }^{\circ} \mathrm{C}$ overnight with the following primary antibodies: anti-LC3 (1:100, Sigma, Germany), anti-p62 (1:100, Santa Cruz, USA) and anti-Beclin1 (1:100, Abcam, UK). After rinsing with PBS, sections were incubated with secondary antibody (biotinylated secondary goat anti-rabbit antibody; 1:200, VECTASTAIN, Vector Laboratories, USA) for $30 \mathrm{~min}$. For color development, sections were treated with a mixture of $0.05 \%$ 3,3-diaminobenzidine containing $0.01 \%$ $\mathrm{H}_{2} \mathrm{O}_{2}$ at $20-23^{\circ} \mathrm{C}$ until the brown color was visible and then washed with TBS, followed by washing with tap water for $10 \mathrm{~min}$. For clearing, sections were treated with alcohol and xylene. Finally, stained sections were examined using light microscope (Nikon eclipse 80i, Nikon Corporation, Japan) at $400 \times$ magnification. Images were captured at 5 different places of renal cortex, outer and inner stripes of outer medulla and inner medullary regions, and LC3, p62 and Beclin1 positive staining were evaluated.

\section{Statistical analysis}

All data were expressed as means \pm standard deviation. Statistical analysis was performed with $t$-test and Microsoft Excel 2016 (Microsoft, USA) was used as the statistical software. The $p$ values less than 0.05 were considered statistically significant.

\section{Results}

\section{Examination of blood parameters}

Serum BUN and Cr levels were measured in both control and cisplatin treated rats after 5 days. The results exhibited a significant $(p<0.001)$ increase in both BUN and Cr levels in the Cis-5D group compared with that in the control group (Fig. 1). The elevated BUN and Cr levels indicated that cisplatin can induce nephrotoxicity, thereby causing renal injury.

\section{Evaluation of renal histological changes}

The histological alterations in the kidney tissues of control 
(A)

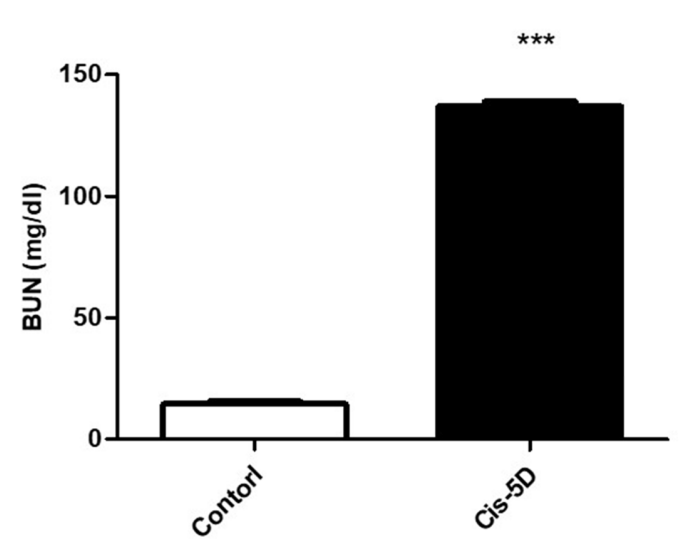

(B)

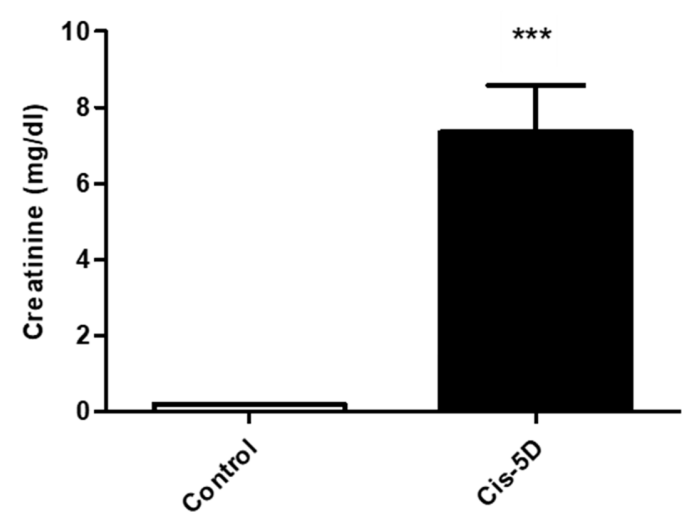

Fig. 1. Effect of cisplatin on blood serum parameters. Blood samples were obtained from the retro-orbital plexus of rats before and after cisplatin treatment $(5 \mathrm{mg} / \mathrm{kg})$, except for the control group, and rats in the cisplatin-treated (Cis-5D) group were sacrificed after 5 days. (A) BUN. (B) creatinine ${ }^{* * *} p<$ 0.001 versus control group.

BUN, blood urea nitrogen.

and cisplatin-treated rats are shown in Fig. 2. No histological changes were observed in the control group compared with those in the cisplatin-treated group (Fig. 2A). However, as shown in Fig. 2B, administration of cisplatin caused severe and widespread necrosis in the proximal tubules, which is a serious damage to the renal structure. In Fig. 2B, there are features of tubular necrosis including patchy denudation of tubular cells with loss of brush border (blue arrow), tubular dilation (black arrow), and intratubular cast formation (green arrow). Furthermore, intratubular obstruction occurred due to the denuded epithelium and cellular debris (yellow arrow). These results indicate that cisplatin treatment can induce kidney injury especially in proximal convoluted tubule due to its toxicity.

IHC analysis of expression of autophagy related-proteins IHC was performed to determine whether autophagy plays

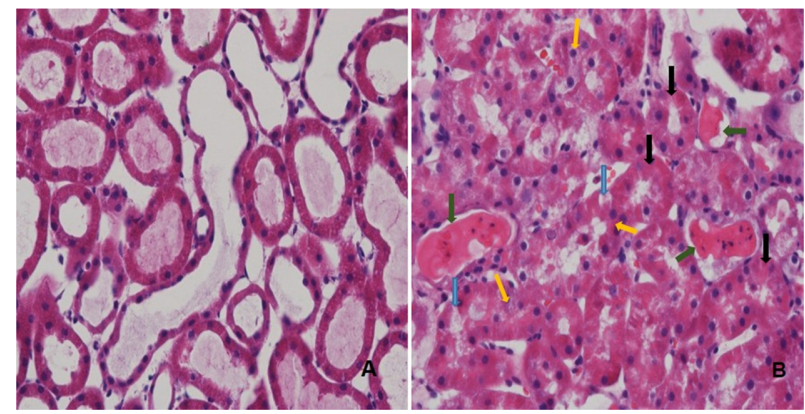

Fig. 2. Photomicrograph of histological alterations in the control and cisplatin-treated Sprague Dawley rats. After intraperitoneal injection of cisplatin $(5 \mathrm{mg} / \mathrm{kg})$, except for the control group, rats in the Cis-5D group were sacrificed after 5 days. Kidney samples were fixed in formalin before routine processing and wax embedding. Sections of kidney were stained with hematoxylin and eosin and were examined by light microscopy with focus on renal tubules to evaluate histological injury. (A) Control group. (B) Cis-5D group. Blue arrows, patchy denudation of tubular cells; black arrows, tubular dilation; green arrows, intratubular cast formation; and yellow arrows, tubular obstruction. Magnification $400 \times$.

Table 1. LC3-positive staining in main divisions of renal structure

\begin{tabular}{lcc}
\hline \hline \multirow{2}{*}{ Renal division } & \multicolumn{2}{c}{ LC3-positive staining } \\
\cline { 2 - 3 } & Control & Cis-5D \\
\hline Glomerulus & - & - \\
Proximal tubule & ++ & ++++ \\
Intermediate tubule & - & - \\
Distal tubule & + & + \\
Collecting system & + & + \\
\hline
\end{tabular}

LC3, light-clain 3; +, LC3 positive staining; -, LC3 negative staining.

a role in cisplatin-induced nephrotoxicity and to determine its localization. In this study, microtubule-associated protein 1 LC3, p62, and Beclin1, the primary markers of autophagy, were evaluated in both control and cisplatin-treated SD rats. Localization of autophagy process was examined in both cortex and medulla in 4 regions viz, cortex, outer stripe and inner stripe of outer medulla, and inner medulla focusing on both renal corpuscles and uriniferous tubules.

As shown in Fig. 3 and Table 1, moderate positive expression of LC3 was observed in both the cortex and outer stripe of outer medulla regions of control rats, whereas strong positive expression of LC3 was observed in the cortex and outer stripe of outer medulla regions of cisplatin-treated rats. Further, renal tubules associated with these 2 regions, such as proximal convoluted and proximal straight tubules exhibited outstanding positive LC3 expression than the distal convoluted tubule and cortical collecting duct, whereas no LC3 expression was observed in glomerulus. However, no LC3 expression was observed in the regions of inner stripe of 


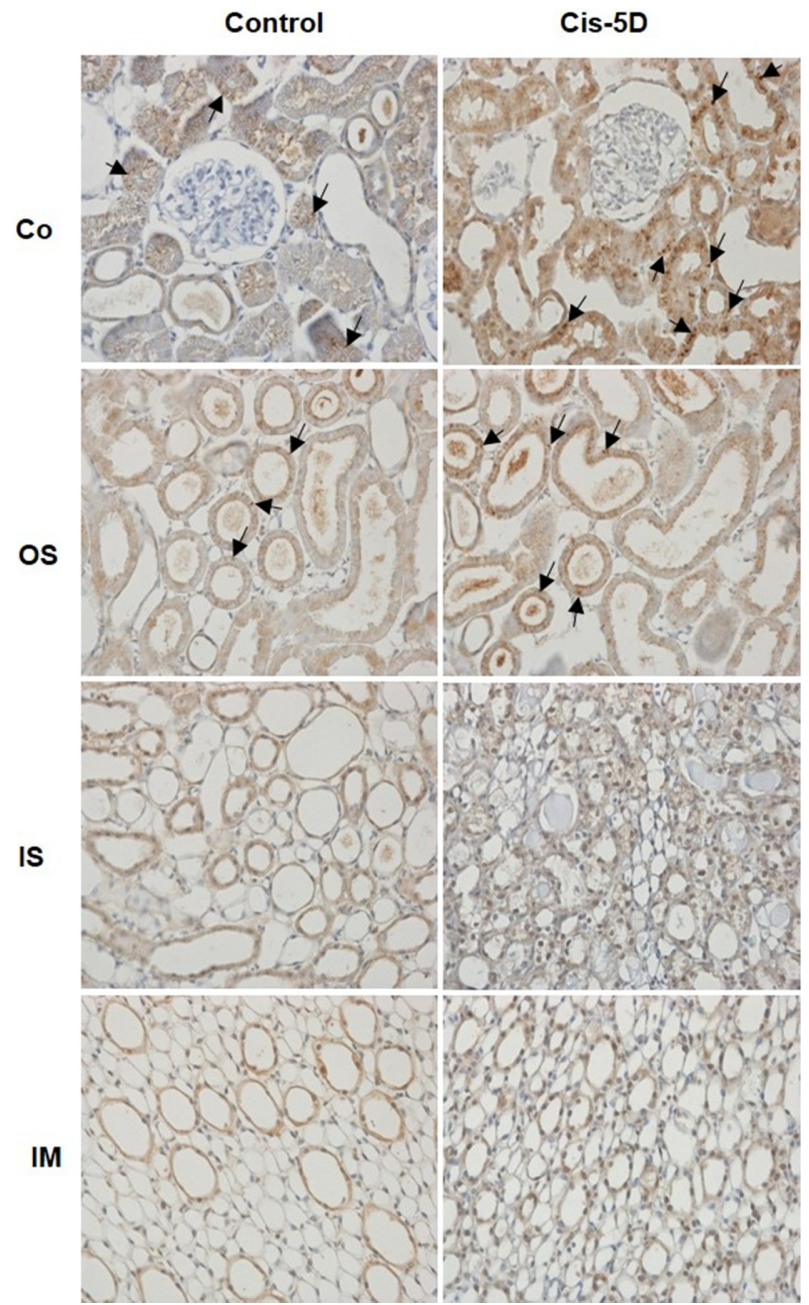

Fig. 3. Immunohistochemical staining of LC3, a key marker of autophagy in rat kidney. After intraperitoneal injection of cisplatin $(5 \mathrm{mg} / \mathrm{kg})$, except for the control group, rats in the Cis-5D group were sacrificed after 5 days. Kidney samples were fixed in formalin before routine processing and wax embedding. Sections of kidney were stained for LC3 and were examined by light microscopy with focus on both renal cortex and medulla regions to determine autophagy localization. Black arrows, LC3 expression. Magnification $400 \times$.

Co, renal cortex; OS, outer stripe of outer medulla; IS, inner stripe of outer medulla; IM, inner medulla; LC3, light-clain 3.

outer medulla and inner medulla in both the control and Cis5D groups. Therefore, uriniferous tubules associated in areas such as distal straight tubule, outer medullary collecting duct, descending thin limb and ascending thin limb (intermediate tubule) and inner medullary collecting duct were not involved in the expression of LC3. These results suggest that cisplatin treatment has a potential to vigorously induce autophagy in proximal tubules in renal cortex and outer medulla regions. Furthermore, our investigation proved that under normal conditions, cellular autophagy proceeds in the proximal tubule in renal cortex and outer medulla of rat kidney.

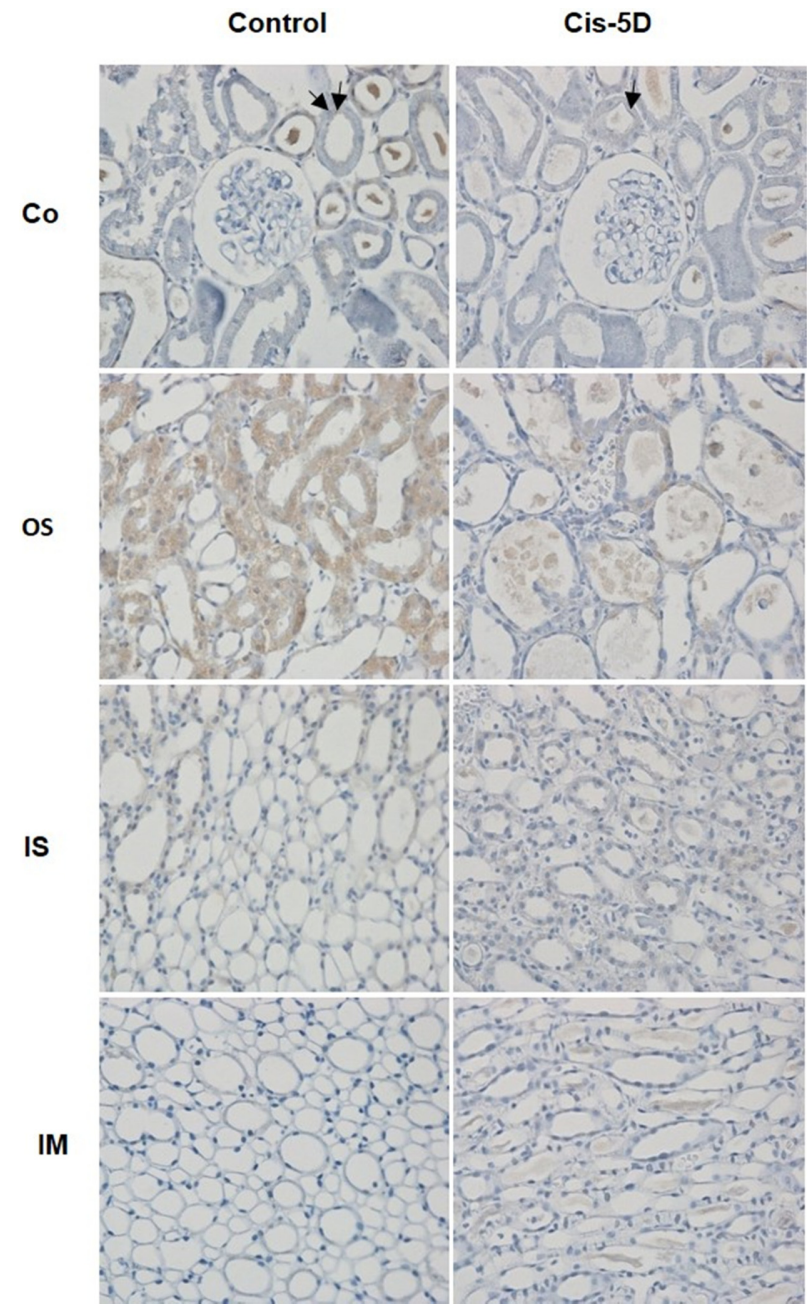

Fig. 4. Immunohistochemical staining of p62, an authoritative marker of autophagy in rat kidney. After intraperitoneal injection of cisplatin $(5 \mathrm{mg} / \mathrm{kg})$, except for the control group, rats in the Cis-5D group were sacrificed after 5 days. Kidney samples were fixed in formalin before routine processing and wax embedding. Sections of kidney were stained for p62 and were examined by light microscopy with focus on both renal cortex and medulla regions to determine the autophagy localization. Black arrows, p62 expression. Magnification $400 \times$.

Co, renal cortex; OS, outer stripe of outer medulla; IS, inner stripe of outer medulla; IM, inner medulla.

p62 is an authoritative receptor of autophagy located throughout the cell. Fig. 4 represents the expression of p62 in the control and cisplatin-treated rats. Contrary to LC3 expression, p62 exhibited minor positive expression in both the control and Cis-5D groups in the region of renal cortex. However, no p62 expression was observed in both outer medulla and inner medulla of the kidney tissue. Moreover, p62 expression was observed only in proximal convoluted tubule cells in the kidney cortex (Table 2). These finding suggest that p62 expression is contrary to LC3 protein expression and exhibits minor distribution, but is localized 
Table 2. p62-positive staining in main divisions of renal structure

\begin{tabular}{lcc}
\hline \hline \multirow{2}{*}{ Renal division } & \multicolumn{2}{c}{ p62-positive staining } \\
\cline { 2 - 3 } & Control & Cis-5D \\
\hline Glomerulus & - & - \\
Proximal tubule & + & + \\
Intermediate tubule & - & - \\
Distal tubule & - & - \\
Collecting system & - & - \\
\hline
\end{tabular}

+, p62 positive staining; -, p62 negative staining.

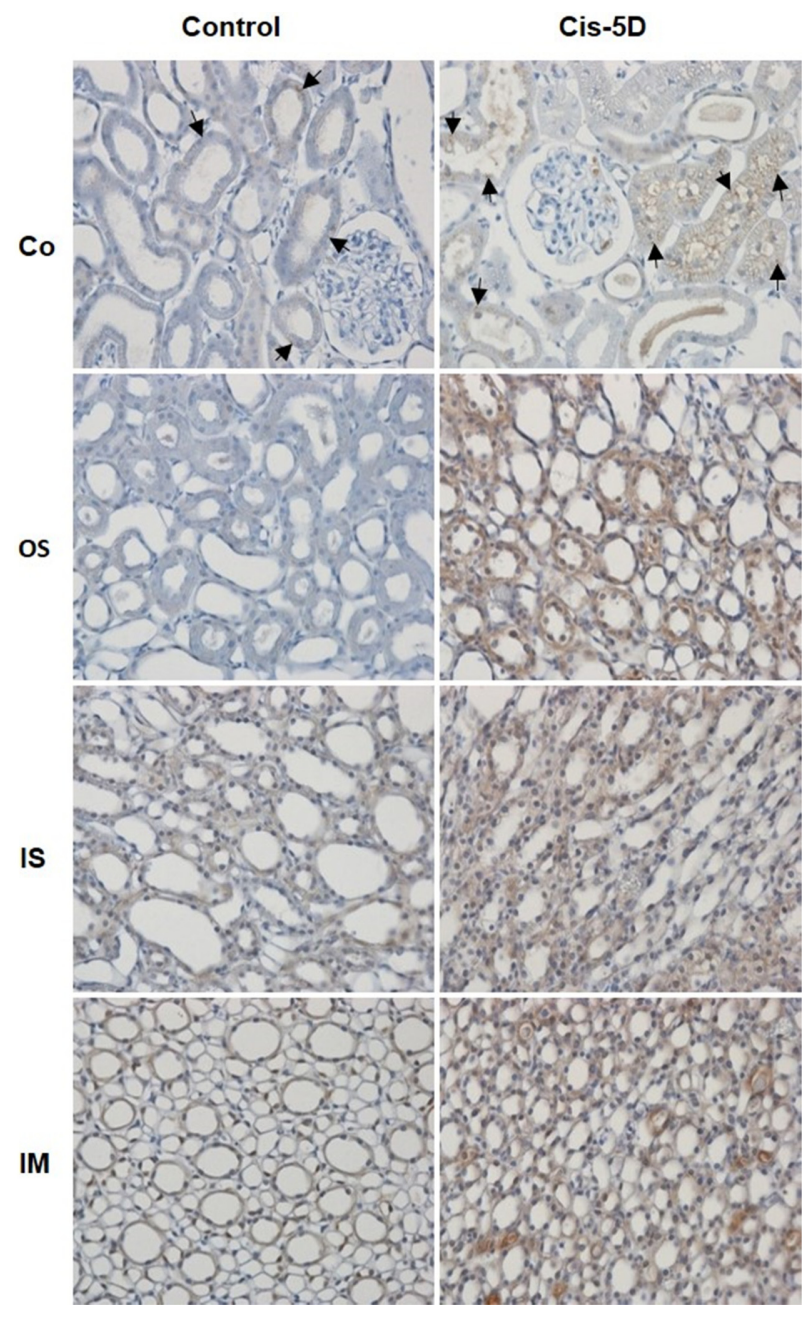

Fig. 5. Immunohistochemical staining of Beclin1, an autophagy marker, in rat kidney. After intraperitoneal injection of cisplatin $(5 \mathrm{mg} / \mathrm{kg})$, except for the control group, rats in the Cis-5D group were sacrificed after 5 days. Kidney samples were fixed in formalin before routine processing and wax embedding. Sections of kidney were stained for Beclin1 and were examined by light microscopy with focus on both renal cortex and medulla regions to determine autophagy localization. Black arrows, Beclin1 expression. Magnification $400 \times$.

Co, renal cortex; OS, outer stripe of outer medulla; IS, inner stripe of outer medulla; IM, inner medulla.
Table 3. Beclin1-positive staining in main divisions of renal structure

\begin{tabular}{lcc}
\hline \hline \multirow{2}{*}{ Renal division } & \multicolumn{2}{c}{ Beclin1-positive staining } \\
\cline { 2 - 3 } \multicolumn{1}{c}{ Control } & Cis-5D \\
\hline Glomerulus & - & - \\
Proximal tubule & + & ++ \\
Intermediate tubule & - & - \\
Distal tubule & - & - \\
Collecting system & - & - \\
\hline
\end{tabular}

+, Beclin1 positive staining; -, Beclin1 negative staining.

only in the proximal convoluted tubule cells.

To study the involvement of autophagy in nephrotoxicity, distribution of Beclin1 was also examined. As shown in Fig. 5, Similar to LC3, Beclin1 also showed moderate positive expression in the control group, whereas strong positive expression was observed in renal cortex of the cisplatininduced nephrotoxicity group. However, no Beclin1 expression was observed in both outer and inner medulla regions. In addition, similar to p62, distribution of Beclin1 was restricted to proximal convoluted tubular cells in the kidney cortex, whereas glomerulus and all other renal tubules were not involved in the expression of these proteins (Table 3). Together, the IHC results proved that autophagy was induced by cisplatin treatment and was localized mainly to the proximal convoluted tubule in the renal cortex and proximal straight tubule in the outer stripe of outer medulla regions.

\section{Discussion}

The nephrotoxicity of cisplatin has been identified since its approval for clinical use over 35 years ago, and despite the toxic effect of cisplatin, it is still widely used in the treatment of various types of cancers. Cisplatin nephrotoxicity exhibits various types of symptoms, such as AKI, hypomagnesemia, distal renal tubular acidosis, renal salt wasting, and hyperuricemia [17]. Pathophysiologically, cisplatin-induced renal injury can be classified into 4 types, viz, tubular toxicity, vascular damage, and glomerular and interstitial injury. In this study, we administered SD rats with cisplatin and found that it had the potential to cause nephrotoxicity, thereby inducing AKI. In line with the previous report [18], rats in our experiment were sacrificed 5 day after cisplatin treatment as cisplatin-induced nephrotoxicity started to develop 3 days post-treatment of cisplatin. Further, we focused on the activation and localization of autophagy in the renal structure under cisplatin-induced stress condition.

Our investigation demonstrated cisplatin-induced renal abnormality in terms of renal function and histological changes in rats. Consistent with previous reports, our analysis showed elevated serum BUN and Cr levels, which indicates cisplatininduced AKI in rats [19]. Our histological examination focused on renal corpuscles and uriniferous tubules in the cortex area and we found severe and widespread necrosis 
associated with renal tubules especially in the proximal convoluted tubule in the cisplatin-treated rats. Furthermore, histology of both superficial and juxtamedullary nephrons was identical, indicating that toxicity was distributed evenly over the cortex region. Cisplatin is an uncharged low weight molecule that is freely filtered through the glomeruli to the renal tubular cells and reaches its highest gradient in the proximal tubular inner medulla and outer cortex. Therefore, these areas are the dominant sites for cisplatin-induced renal injury and cause injury to other structures, such as distal and collecting tubules [20].

Moreover, our investigation concentrated on the subcellular localization and redistribution of autophagy markers; autophagosome staining was performed by IHC. The results revealed elevated expression of LC 3 and Beclin1 and decreased expression of p62 under cisplatin nephrotoxicity. These autophagy markers indicated that cellular autophagy was rapidly induced in the kidney tissue after cisplatin treatment. Furthermore, induction of autophagy provides protection against proximal tubular cell injury, and the expression of the aforementioned proteins was restricted to the renal cortex and outer medulla regions. Moreover, our results also revealed that under cisplatin stress and normal condition, autophagy proceeds at a considerable rate in the proximal tubules of the above-mentioned kidney areas.

Autophagy is an essential part of different biological processes such as cell growth and homeostasis, via maintaining the balance between the syntheses, degradation, and recycling of cellular components [21]. The basic autophagy machinery consists primarily of 4 steps, viz, autophagy initiation, nucleation, elongation, and closure, and Atg proteins are responsible for autophagy progression [22]. Autophagy is initiated by the formation of the isolation membrane, known as phagophore that contains cytoplasmic components. Expansion of the isolation membrane, known as vesical elongation, leads to the formation of autophagosome [23]. During elongation to closure, LC3 (the mammalian ortholog of Atg8) conjugation reaction is stimulated by the Atg 12-Atg5 complex. LC3-I is catalyzed by Atg7 and Atg3 to form LC3II, which is a key requirement for autophagosomes [24,25]. Finally, the outer membrane of autophagosomes fuses with the lysosomes to form autolysosomes, which are responsible for recycling and degradation of the sequestered materials. p62 is also localized to autophagosomes on interaction with LC3, and aggregation of p62 is a characteristic of autophagydeficient cells [26,27]. Therefore, impaired autophagy evidenced by accumulation of p62 in the cytoplasm of renal cells is because of the autophagy-lysosomal degradation pathway.

Beclin1, the mammalian ortholog of yeast 6 , is involved in vesicle nucleation and early event of autophagosome formation [28] and stimulates lipid kinase activity, which is important for the recruitment of autophagy proteins to the sites of autophagosome formation [29]. In addition, Beclin1 interacts with anti-apoptotic Bcl-2 family members, and this interaction suppresses Beclin1 from accumulating in the initial autophagosomal structure, thereby attenuating autophagy [30]. The expression level of Beclin1 and Bcl-2 are the major determinants of whether cells undergo autophagy or apoptosis during tumorigenesis and chemotherapy [31]. We confirmed increased expression of Beclin1 by IHC, thereby confirming induced cellular autophagy in renal proximal tubular cells. Based on IHC results, this study demonstrated the types of renal cells involved in autophagy for protection against cisplatin-induced nephrotoxicity.

To conclude, our study evidenced that chemotherapy using cisplatin can cause nephrotoxicity, thereby inducing AKI. Under this stressful condition, the regular autophagy mechanism in proximal tubular cells is accelerated to provide defense against AKI by modulating several deteriorative pathways. Therefore, treatment with cisplatin may accelerate the autophagy mechanism under the regulation of LC3, p62, and Beclin1 in the proximal tubular cells of renal cortex and outer medulla regions. Therefore, increase autophagy and its associated pathways may possible therapeutic intervention in AKI.

\section{Acknowledgments}

This work was supported by the research fund of Chungnam National University.

\section{References}

1. Oh GS, Kim HJ, Shen A, Lee SB, Khadka D, Pandit A, So HS. Cisplatin-induced kidney dysfunction and perspectives on improving treatment strategies. Electrolyte Blood Press 2014; 12:55-65.

2. Yao X, Panichpisal K, Kurtzman N, Nugent K. Cisplatin nephrotoxicity: a review. Am J Med Sci 2007;334:115-124.

3. Bonventre JV, Weinberg JM. Recent advances in the pathophysiology of ischemic acute renal failure. J Am Soc Nephrol 2003;14:2199-2210.

4. Devarajan P. Update on mechanisms of ischemic acute kidney injury. J Am Soc Nephrol 2006;17:1503-1520.

5. Goligorsky MS. Whispers and shouts in the pathogenesis of acute renal ischaemia. Nephrol Dial Transplant 2005;20:261266.

6. Molitoris BA, Sutton TA. Endothelial injury and dysfunction: role in the extension phase of acute renal failure. Kidney Int 2004;66:496-499.

7. Vaidya VS, Ferguson MA, Bonventre JV. Biomarkers of acute kidney injury. Annu Rev Pharmacol Toxicol 2008;48: 463-493.

8. Sastry J, Kellie SJ. Severe neurotoxicity, ototoxicity and nephrotoxicity following high-dose cisplatin and amifostine. Pediatr Hematol Oncol 2005;22:441-445.

9. Pfeifer U, Guder WG. Stimulation of cellular autophagy by parathyroid hormone and cyclic adenosine $3^{\prime}, 5^{\prime}$ : monophosphate in isolated tubular fragments from the rat's kidney cortex. Virchows Arch B Cell Pathol Incl Mol Pathol 1975;19:51-67.

10. Pfeifer U, Scheller H. A morphometric study of cellular autophagy including diurnal variations in kidney tubules of 
normal rats. J Cell Biol 1975;64:608-621.

11. Livingston MJ, Dong Z. Autophagy in acute kidney injury. Semin Nephrol 2014;34:17-26.

12. Bolisetty S, Traylor AM, Kim J, Joseph R, Ricart K, Landar A, Agarwal A. Heme oxygenase-1 inhibits renal tubular macroautophagy in acute kidney injury. J Am Soc Nephrol 2010;21:1702-1712.

13. Jiang M, Wei Q, Dong G, Komatsu M, Su Y, Dong Z. Autophagy in proximal tubules protects against acute kidney injury. Kidney Int 2012;82:1271-1283.

14. Periyasamy-Thandavan S, Jiang M, Wei Q, Smith R, Yin $\mathrm{XM}$, Dong Z. Autophagy is cytoprotective during cisplatin injury of renal proximal tubular cells. Kidney Int 2008;74: 631-640.

15. Brady HR, Kone BC, Stromski ME, Zeidel ML, Giebisch G, Gullans SR. Mitochondrial injury: an early event in cisplatin toxicity to renal proximal tubules. Am J Physiol 1990;258: F1181-F1187.

16. Gordon JA, Gattone VH 2nd. Mitochondrial alterations in cisplatin-induced acute renal failure. Am J Physiol 1986;250: F991-F998.

17. Miller RP, Tadagavadi RK, Ramesh G, Reeves WB. Mechanisms of Cisplatin nephrotoxicity. Toxins (Basel) 2010;2:2490-2518.

18. Pezeshki Z, Khosravi A, Nekuei M, Khoshnood S, Zandi E, Eslamian M, Talebi A, Emami SN, Nematbakhsh M. Time course of cisplatin-induced nephrotoxicity and hepatotoxicity. J Nephropathol 2017;6:163-167.

19. Liu Q, Hu S, He Y, Zhang J, Zeng X, Gong F, Liang L. The protective effects of Zhen-Wu-Tang against cisplatininduced acute kidney injury in rats. PLoS One 2017;12: e0179137.

20. Kuhlmann MK, Burkhardt G, Köhler H. Insights into potential cellular mechanisms of cisplatin nephrotoxicity and their clinical application. Nephrol Dial Transplant 1997;12: 2478-2480.

21. Mizushima N, Ohsumi Y, Yoshimori T. Autophagosome formation in mammalian cells. Cell Struct Funct 2002;27: 421-429.

22. Tanaka Y, Kume S, Kitada M, Kanasaki K, Uzu T, Maegawa H, Koya D. Autophagy as a therapeutic target in diabetic nephropathy. Exp Diabetes Res 2012;2012:628978.

23. Hamasaki M, Furuta N, Matsuda A, Nezu A, Yamamoto A, Fujita N, Oomori H, Noda T, Haraguchi T, Hiraoka Y, Amano A, Yoshimori T. Autophagosomes form at ER-mitochondria contact sites. Nature 2013;495:389-393.

24. Nakatogawa H, Suzuki K, Kamada Y, Ohsumi Y. Dynamics and diversity in autophagy mechanisms: lessons from yeast. Nat Rev Mol Cell Biol 2009;10:458-467.

25. Ravikumar B, Sarkar S, Davies JE, Futter M, GarciaArencibia M, Green-Thompson ZW, Jimenez-Sanchez M, Korolchuk VI, Lichtenberg M, Luo S, Massey DC, Menzies FM, Moreau K, Narayanan U, Renna M, Siddiqi FH, Underwood BR, Winslow AR, Rubinsztein DC. Regulation of mammalian autophagy in physiology and pathophysiology. Physiol Rev 2010;90:1383-1435.

26. Bjørkøy G, Lamark T, Brech A, Outzen H, Perander M, Overvatn A, Stenmark H, Johansen T. p62/SQSTM1 forms protein aggregates degraded by autophagy and has a protective effect on huntingtin-induced cell death. J Cell Biol 2005; 171:603-614.

27. Komatsu M, Waguri S, Koike M, Sou YS, Ueno T, Hara T, Mizushima N, Iwata J, Ezaki J, Murata S, Hamazaki J, Nishito Y, Iemura S, Natsume T, Yanagawa T, Uwayama J, Warabi E, Yoshida H, Ishii T, Kobayashi A, Yamamoto M, Yue Z, Uchiyama Y, Kominami E, Tanaka K. Homeostatic levels of p62 control cytoplasmic inclusion body formation in autophagy-deficient mice. Cell 2007;131:1149-1163.

28. Liang XH, Jackson S, Seaman M, Brown K, Kempkes B, Hibshoosh H, Levine B. Induction of autophagy and inhibition of tumorigenesis by Beclin 1. Nature 1999;402:672-676.

29. Rubinstein $\mathrm{AD}$, Kimchi A. Life in the balance - a mechanistic view of the crosstalk between autophagy and apoptosis. J Cell Sci 2012;125:5259-5268.

30. Liang XH, Kleeman LK, Jiang HH, Gordon G, Goldman JE, Berry G, Herman B, Levine B. Protection against fatal Sindbis virus encephalitis by Beclin, a novel Bcl-2-interacting protein. J Virol 1998;72:8586-8596.

31. Kang R, Zeh HJ, Lotze MT, Tang D. The Beclin 1 network regulates autophagy and apoptosis. Cell Death Differ 2011; 18:571-580. 